\title{
Successful closure of a tracheocutaneous fistula after tracheostomy using two skin flaps: a case report
}

\author{
Yui Watanabe ${ }^{1 *}$, Tadashi Umehara' ${ }^{1}$ Aya Harada' ${ }^{1}$ Masaya Aoki ${ }^{1}$, Takuya Tokunaga', Soichi Suzuki ${ }^{1}$, Go Kamimura', \\ Kazuhiro Wakida', Toshiyuki Nagata', Tsunayuki Otsuka', Naoya Yokomakura' ', Kota Kariatsumari', Yoshihiro Nakamura', \\ Yuko Watanabe ${ }^{2}$ and Masami Sato ${ }^{1}$
}

\begin{abstract}
A tracheocutaneous fistula may develop when a tracheostomy orifice epithelializes during a prolonged course of healing or undernutrition. Various techniques for closing such fistulae have been reported. However, a standard procedure has not yet been established. We, herein, present a case involving a 35-year-old woman who developed a tracheocutaneous fistula after tracheostomy. We closed the fistula using two skin flaps to cover the tracheal lumen and skin defect, respectively. The advantage of this technique is that it allows the tracheal lumen to be covered by inversed skin epithelium and ensures that the suture line of the skin does not match up with that of the subcutaneous tissue.
\end{abstract}

Keywords: Tracheocutaneous fistula; Tracheostomy; Skin flap

\section{Background}

Tracheostomy is a general surgical procedure performed by many thoracic surgeons on a routine basis. A tracheostomy orifice closes by second intention in many routine cases. However, the orifice sometimes epithelializes and develops a tracheocutaneous fistula, especially if the healing course is prolonged or the patient has a poor nutritional status. In such cases, surgery is required to close the fistula. During surgery, it is important to definitively cover both the tracheal and skin defects. Additionally, attention should be paid to avoid promoting postoperative tracheal stenosis secondary to granulation.

\section{Case presentation}

A 35-year-old woman was scheduled to undergo surgery to close her tracheocutaneous fistula. The patient had previously undergone tracheostomy with an inverted Ushaped tracheal incision and mechanical ventilation for central neurogenic respiratory failure due to autoimmune

\footnotetext{
* Correspondence: yuiw@mac.com

'Department of General Thoracic Surgery, Graduate School of Medical and Dental Sciences, Kagoshima University, 8-35-1 Sakuragaoka, Kagoshima 890-8570, Japan

Full list of author information is available at the end of the article
}

limbic encephalitis when she was 30 years old. Although she recovered from her previous condition after 11 months of tracheal cannulation, she subsequently developed a tracheocutaneous fistula due to the prolonged course of healing and poor nutritional status. Laboratory analysis revealed that her white blood cell count was $2900 / \mu \mathrm{L}$, serum total protein level was $5.3 \mathrm{~g} / \mathrm{dL}$, and serum albumin level was $3.0 \mathrm{~g} / \mathrm{dL}$. Neck sagittal computed tomography showed a tracheocutaneous fistula (Fig. 1a). Although repair of such fistulae is possible under local anesthesia, her surgery was performed under general anesthesia because the encephalitis had caused residual restlessness.

The skin incision was designed as a box around the fistula with two sequential straight incisions emanating from one corner (Fig. 1b). The length of each skin incision was $25 \mathrm{~mm}$. Bilateral hinge flaps to close the fistula were created from the left and right sides of the tracheal defect, and superfluous skin from each hinge flap was trimmed (Fig. 2a). A single flap covering the skin defect was elevated from the lateral aspect of the right hinge flap to join with the corresponding sides of the defect (Fig. 2b). The hinge flaps were inversed to cover the tracheal lumen with skin epithelium and sutured tightly 

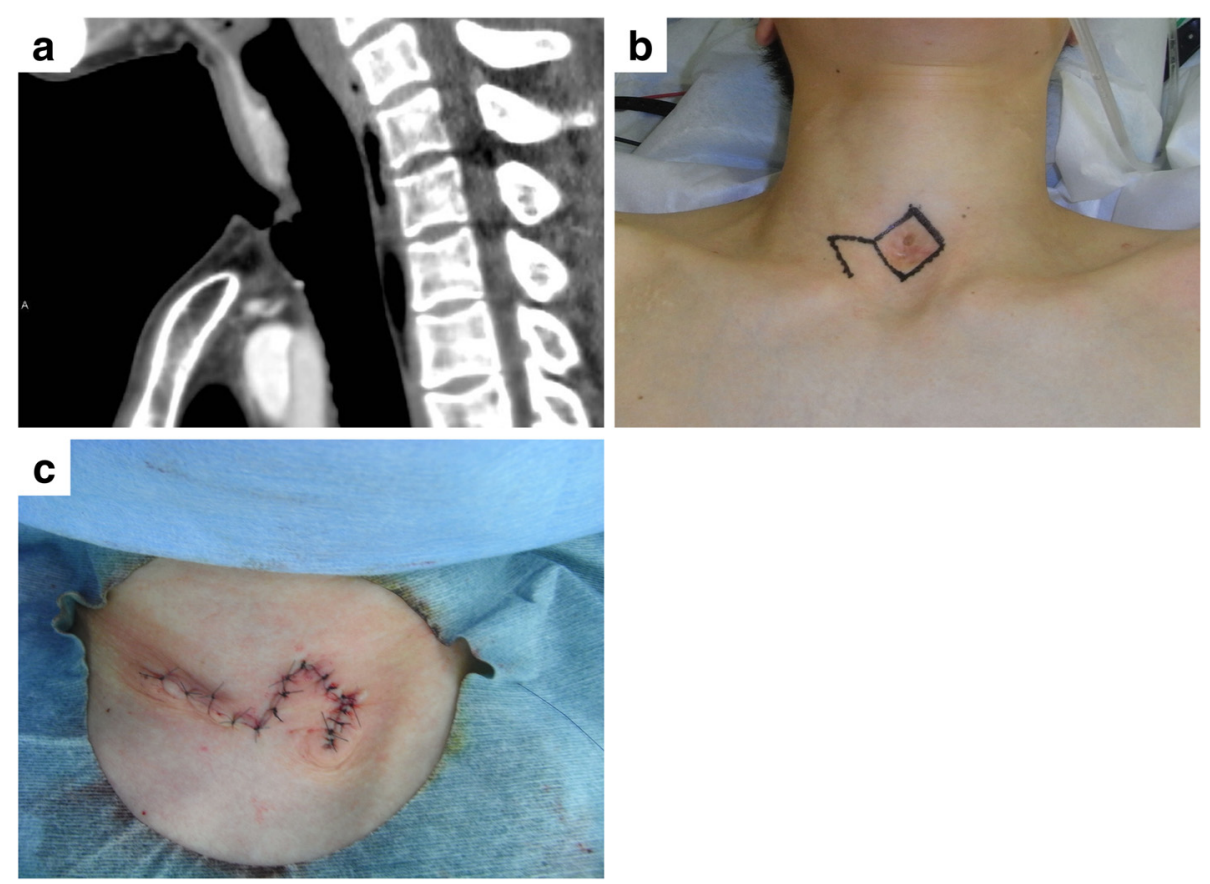

Fig. 1 Preoperative and operative findings. a Cervical sagittal computed tomography showed a tracheocutaneous fistula. b The skin incision was designed as a box around the fistula and two sequential straight lines. c A single flap was elevated and rotated to cover the skin defect, and the skin was sutured layer by layer

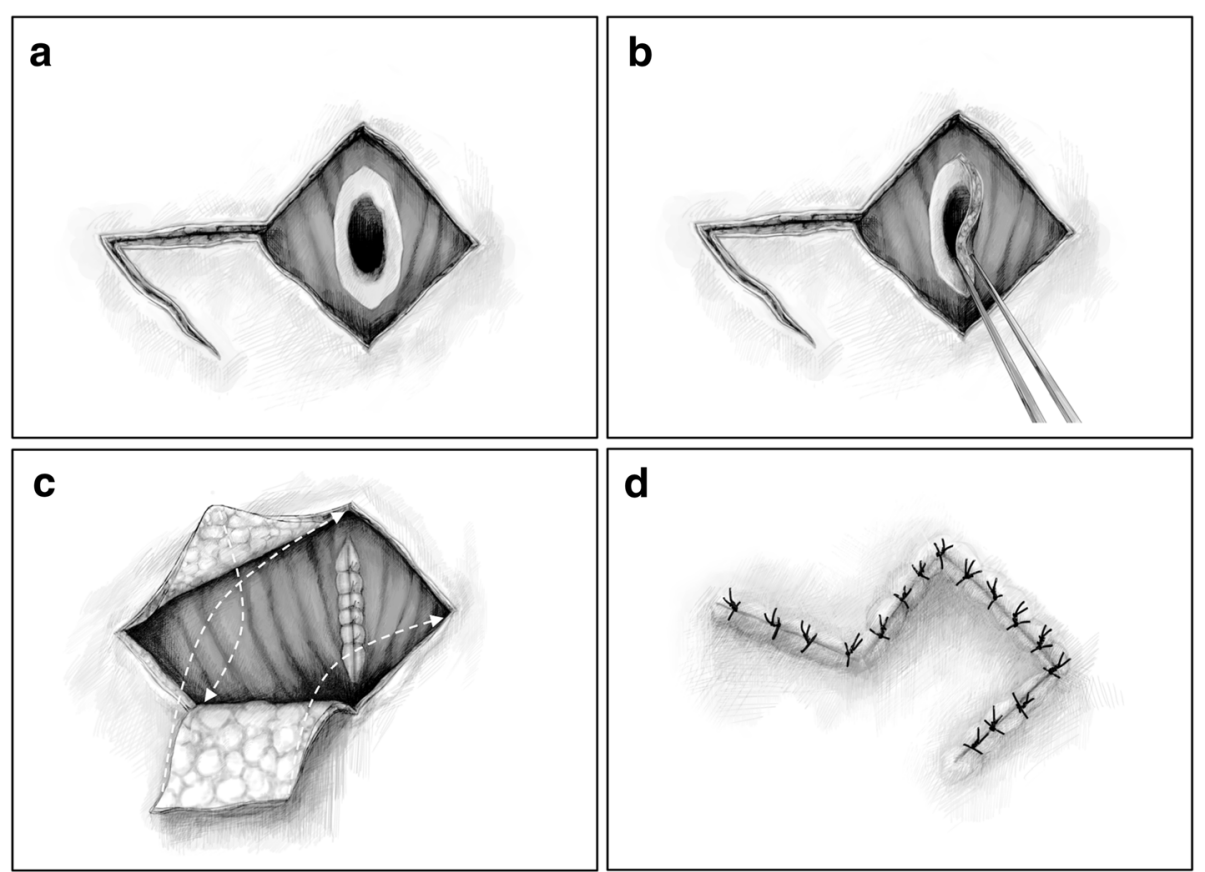

Fig. 2 Operative schemes. a Bilateral hinge flaps were created to close the fistula. The flaps were taken from the left and right sides of the tracheal defect, and the superfluous skin of each hinge flap was trimmed. $\mathbf{b}$ The hinge flaps were inversed to cover the tracheal lumen with skin epithelium and sutured tightly with the platysma muscle. c A single flap to cover the skin defect was elevated from the lateral aspect of the right hinge flaps. The arrows indicate how the skin flaps were positioned. $\mathbf{d}$ The single flap was elevated and rotated to cover the skin defect, and the skin was sutured layer by layer 
with the platysma muscle using absorbent strings (Fig. 2b). The elevated and rotated single flap covered the skin defect, and nonabsorbent suture was used to close the skin in a layer-by-layer fashion to avoid creating dead space (Figs. 1c and 2c). The operative time was $58 \mathrm{~min}$, and a drainage tube was not placed. Her postoperative course was uneventful, and she had no recurrence of the tracheocutaneous fistula for 18 months.

Several procedures to close tracheocutaneous fistulae have been previously reported [1-7]. However, no standard, easily performed procedure has been established worldwide. One advantage of the procedure described herein is that postoperative tracheal stenosis secondary to granulation is not likely to occur because the tracheal lumen is covered by inversed skin epithelium. The bilateral hinge flaps closing the tracheal defect can be lifted from not only the left and right sides but also from the upper and lower sides depending on the form of the defect. Moreover, the superfluous skin of each hinge flap can be trimmed to create appropriately sized flaps. Another advantage is that recurrence of the tracheocutaneous fistula after wound dehiscence is not likely to occur because the suture line of the skin cannot overlap with that of the subcutaneous tissue. Most patients can undergo this procedure with local anesthesia. This technique for closing tracheocutaneous fistulae after tracheostomy using two skin flaps is thus considered to be beneficial.

\section{Conclusions}

We have presented a case of successful closure of a tracheocutaneous fistula after tracheostomy using two skin flaps. This technique has two advantages: (1) it allows the tracheal lumen to be covered by inversed epithelium and (2) it ensures that the suture line of the skin does not match up with that of the subcutaneous tissue.

\section{Consent}

Written informed consent was obtained from the patient for publication of this case report and any accompanying images. A copy of the written consent is available for review by the Editor-in-Chief of this journal.

\section{Abbreviation \\ $\mathrm{CT}$ : computed tomography.}

\section{Competing interests}

The authors declare that they have no competing interests.

\section{Authors' contributions}

YW, TU, YKW, and MS participated in the conception, design, and analysis of this case report and drafted the manuscript. AH, MA, TT, SS, GK, KW, TN, TO, $N Y, K K$, and YN participated in its design and coordination and helped to draft the manuscript. All authors read and approved the final manuscript.

\section{Acknowledgements}

The authors would like to express their gratitude to Mr. Shin Saito, Ms. Akiko Nibe, and their colleagues of TSUBASA Co., Ltd., Akita, Japan, for their excellent illustrations.

\section{Author details}

${ }^{1}$ Department of General Thoracic Surgery, Graduate School of Medical and Dental Sciences, Kagoshima University, 8-35-1 Sakuragaoka, Kagoshima 890-8570, Japan. ${ }^{2}$ Department of Anesthesiology and Critical Care Medicine, Graduate School of Medical and Dental Sciences, Kagoshima University, Kagoshima, Japan.

Received: 9 February 2015 Accepted: 22 May 2015

Published online: 27 May 2015

\section{References}

1. Kamiyoshihara M, Nagashima T, Takeyoshi I. A novel technique for closing a tracheocutaneous fistula using a hinged skin flap. Surg Today. 2011;41:1166-8.

2. Schroeder Jr JW, Greene RM, Holinger LD. Primary closure of persistent tracheocutaneous fistula in pediatric patients. J Pediatr Surg. 2008;43:1786-90.

3. Sautter NB, Krakovitz PR, Solares CA, Koltai PJ. Closure of persistent tracheocutaneous fistula following "starplasty" tracheostomy in children. Int J Pediatr Otorhinolaryngol. 2006;70:99-105.

4. Eliashar R, Sichel JY, Eliachar I. A new surgical technique for primary closure of long-term tracheostomy. Otolaryngol Head Neck Surg. 2005;132:115-8.

5. Haynes JH, Bagwell CE, Salzberg AM. Management of persistent pediatric tracheostomal fistulas. J Pediatr Surg. 1995;30:566-7.

6. Goldsmith AJ, Abramson AL, Myssiorek D. Closure of tracheocutaneous fistula using a modified cutaneous Z-plasty. Am J Otolaryngol. 1993;14:240-5.

7. Bishop JB, Bostwick J, Nahai F. Persistent tracheostomy stoma. Am J Surg. 1980;140:709-10

\section{Submit your manuscript to a SpringerOpen ${ }^{\circ}$ journal and benefit from:}

- Convenient online submission

- Rigorous peer review

- Immediate publication on acceptance

- Open access: articles freely available online

- High visibility within the field

- Retaining the copyright to your article

Submit your next manuscript at springeropen.com 